\title{
Pioglitazone and metformin fixed-dose combination in type 2 diabetes mellitus: an evidence-based review of its place in therapy
}

\author{
Giuseppe Derosa, Sibilla Anna Teresa Salvadeo
}

Department of Internal Medicine and Therapeutics, University of Pavia, Pavia, Italy

\begin{abstract}
Introduction: Type 2 diabetes mellitus, a metabolic disease with increasing incidence, is one of the most important cardiovascular risk factors. Insulin resistance represents the common mechanism that leads to type 2 diabetes in obese subjects. Metformin and the thiazolidinediones, pioglitazone and rosiglitazone, are insulin-sensitizing agents available for treatment of type 2 diabetes. Large clinical trials have demonstrated the effectiveness of both metformin and pioglitazone in reducing cardiovascular morbidity and mortality. The fixed-dose combination of metformin and pioglitazone appears to be a good option for treating diabetes in insulin-resistant patients.
\end{abstract}

Aims: The purpose of this article is to review the place in therapy of a fixed-dose combination of pioglitazone and metformin in the management of patients with type 2 diabetes.

Evidence review: The current evidence suggests that combined therapy may help to achieve the recommended goals in the management of diabetes. A fixed-dose formulation of pioglitazone and metformin may provide advantages in terms of glycemic control and other cardiovascular risk factors frequently associated with diabetes.

Place in therapy: The current evidence shows that a fixed-dose formulation of pioglitazone and metformin offers an effective option for the management of patients with type 2 diabetes when monotherapy fails in the achievement of the recommended standards of care.

Core Evidence. 2007;2(3):189-198

Key words: cardiovascular risk factors, diabetes, pioglitazone, metformin, outcomes.

\section{Core evidence place in therapy summary for pioglitazone/metformin fixed-dose combination in type 2 diabetes mellitus}

\begin{tabular}{|c|c|c|}
\hline Outcome measure & Evidence & Implications \\
\hline Improvement in glycemic control and insulin sensitivity & Clear & $\begin{array}{l}\text { As effective as pioglitazone/sulfonylureas and metformin/sulfonylureas on glycemic } \\
\text { control. Comparable to rosiglitazone/metformin on insulin sensitivity, and more } \\
\text { effective than metformin/sulfonylureas }\end{array}$ \\
\hline Improvement in lipid abnormalities & Substantial & More effective than rosiglitazone/sulfonylureas combination \\
\hline Improvement in other cardiovascular risk factors & Substantial & Improves nonconventional cardiovascular risk factors \\
\hline Hypertension & Moderate & $\begin{array}{l}\text { More long-term clinical trials adjusted for use of antihypertensive treatment } \\
\text { are required }\end{array}$ \\
\hline Patient convenience & Moderate & Adverse effects comparable to those with other combinations \\
\hline Cost effectiveness & Unclear & More long-term studies required \\
\hline
\end{tabular}




\section{Scope, aims, and objectives}

Pioglitazone/metformin fixed-dose formulation combines two insulin sensitizers: metformin, a biguanide, and pioglitazone, a thiazolidinedione (TZD). This combination has been developed for the management of patients with type 2 diabetes mellitus who are intolerant or not adequately controlled with metformin monotherapy, and has been approved in the US and EU. Combination treatment may be more suitable for some diabetic patients who are frequently treated with multiple drugs.

Metformin and pioglitazone are well-known insulin-sensitizing agents that act through different mechanisms. The mechanism of action of both metformin and pioglitazone is still not completely understood. However, the large spectrum of effects of pioglitazone makes the combination of metformin and pioglitazone a promising treatment option not only for optimizing management of glycemic control but also for prevention of the cardiovascular complications of diabetes. Side effects induced by combination treatment are also addressed in the article. The largest part of literature available for the combination treatment is from studies of pioglitazone and metformin given together as separate drugs, since few data are available with fixed-dose combination treatment.

The purpose of this article is to review the place in therapy of a fixed-dose combination of pioglitazone and metformin in the management of patients with type 2 diabetes.

\section{Methods}

The English language medical literature was reviewed for appropriate articles on pioglitazone/metformin combination treatment for the treatment of type 2 diabetes. The following databases were searched between May and June 2007 using the search term "pioglitazone metformin."

- PubMed, http://www.ncbi.nlm.nih.gov/entrez/query.fcgi

- EMBASE, http://www.datastarweb.com

- Database of Abstracts of Reviews of Effects (DARE), National Health Service (NHS) Economic Evaluation Database (NHSEED), Health Technology Assessment (HTA), http://www.york.ac.uk/inst/crd/crddatabases.htm. All three databases were searched together. All fields searched

- Cochrane Database of Systematic Reviews (CDSR), http://www.cochrane.org

- BIOSIS, http://www.datastarweb.com

- National Institute for Health and Clinical Evidence (NICE), http://www.nice.org.uk

No date limitations were placed on the search. Numerous guidelines related to type 2 diabetes management were examined, although only American Diabetes Association (ADA) guidelines were considered (ADA 2007).

The level of evidence identified from the literature search is described in Table 1.

\section{Table 1 | Evidence base included in the review}

\begin{tabular}{|lcc|}
\hline Category & \multicolumn{2}{c|}{ Number of records } \\
\cline { 2 - 3 } & Full papers & Abstracts $^{\text {a }}$ \\
\hline Initial search & 50 & 23 \\
records excluded & 23 & 22 \\
records included & 27 & 4 \\
Additional records identified & 4 & 0 \\
Level 1 clinical evidence & 4 & 0 \\
(systematic review, meta analysis) & & 3 \\
Level 2 clinical evidence (RCT) & 27 & 0 \\
Level $\geq 3$ clinical evidence & 0 & 1 \\
Economic evidence & 0 & \\
\hline For definition of levels of evidence, see Editorial Information on inside back cover or on \\
Core Evidence website. \\
alncludes two studies on fixed-dose combination.
\end{tabular}

\section{Disease overview}

Increasing numbers of patients with type 2 diabetes are identified every year all over the world; recent data suggest that around 246 million people are affected by type 2 diabetes, and this number is expected to be around 380 million by 2025 (IDF 2006). The associated annual mortality rate has been estimated to be around $5.5 \%$ of all patients (White et al. 2003). The prevalence of type 2 diabetes is largely variable with ethnicity and age. Direct medical costs for diabetes (i.e. the total costs of medical care) are increased when diabetes is poorly controlled and diabetes complications are present (Gilmer et al. 2007).

The underlying mechanism that leads to diabetes is recognized as insulin resistance, which appears to be involved in a number of other cardiovascular disorders such as hypertension (Rosak 2002). Frequently, type 2 diabetes is associated with higher cardiovascular risk (Mann 2002). The role of type 2 diabetes in the development of atherosclerotic disease is well established (National Service Framework for Diabetes 2001). Recently, a cluster of cardiovascular disorders have been unified under the definition of "metabolic syndrome," which is characterized by a high cardiovascular risk (Haffner 2007).

Types 2 diabetes is frequently diagnosed in adults and two conditions-namely impaired glucose tolerance and impaired fasting glucose-are present in people who are at higher risk for developing diabetes. In particular, central (androgen) obesity is directly related to diabetes in genetically susceptible subjects (Beys et al. 2007). 


\section{Current therapy options}

Every year, level 1 evidence and recommendations are published regarding the treatment of type 1 and type 2 diabetes and its complications (e.g. ADA 2007). Diabetes management includes not only blood glucose control, but also treatment of complications and comorbidity control. Diet and physical exercise are fundamental to achieve the goals recommended for glycemic control [glycosylated hemoglobin $\left(\mathrm{HbA}_{1 \mathrm{c}}\right)<7 \%$ ] and reducing low-density lipoprotein cholesterol (LDL-C) ( $\leq 70 \mathrm{mg} / \mathrm{dL})$ (Mann 2002; ADA 2007). Blood pressure recommendations are more restrictive in diabetic patients compared with the healthy population: systolic (SBP) and diastolic (DBP) values of $\leq 130 \mathrm{mmHg}$ and $80 \mathrm{mmHg}$ are suggested in type 2 diabetes. Dyslipidemia is usually recommended to be treated by statins and fibrates (MRC/BHF 1999), and aspirin treatment should always be started in diabetic patients who present with secondary cardiovascular risk factors, such as hypertension (UKPDS 1998; ADA 2007).

Two major classes of drug are currently used for the treatment of diabetes: insulin sensitizers and secretagogs. The first class includes biguanides (primarily metformin) and TZDs (including pioglitazone and rosiglitazone); the second comprises sulfonylureas (SUs) (Kyvik et al. 1995), glinides (Standl \& Fuchtenbusch 2003), and alfa-glucosidase inhibitors such as acarbose and voglibose (Ahmann \& Riddle 2002). Insulin is recommended in type 2 diabetes in nonresponding patients previously treated with oral antidiabetic drugs or in particular situations where diabetes is poorly controlled (e.g. surgery, intercurrent disease, pregnancy, renal impairment) (ADA 2007). Pharmacologic treatment is started when diet and exercise are not sufficient to achieve the therapeutic goal $\mathrm{HbA}_{1 \mathrm{c}}$ reduction to $<7 \%$. Currently, the first choice in obese [body mass index $(\mathrm{BMI})>30 \mathrm{~kg} / \mathrm{m}^{2}$ ] type 2 diabetic patients not controlled with diet and physical activity is metformin, an insulin sensitizer that acts primarily in the liver, thus reducing gluconeogenesis (Takiya \& Chawla 2002). When glycemic control is not achieved with a single drug, combination therapy is recommended (Nathan et al. 2006). The most commonly employed combination in clinical practice, and also the best studied, is metformin and a SU (Krentz \& Bailey 2005). SUs are secretagogs and effectively improve insulin secretion from beta cells of pancreatic islets. The choice of SU is dependent on the presence of a residual insulin secretion. SUs are commonly well tolerated, although some important side effects such as weight gain can occur during treatment (Kyvik et al. 1995). To date, SUs, metformin, and TZDs have been shown to be similarly effective in achieving the recommended goals for glycemic control (Bolen et al. 2007). New drugs are under development and are represented by peptides, amino acids, and peptidomimetics (Jain \& Chawrai 2005).

Recently, both pioglitazone and rosiglitazone have been approved in the US and EU in association with insulin, and the combination of a TZD and metformin has been introduced.

\section{Clinical evidence with fixed-dose pioglitazone/ metformin}

Bioequivalence between the fixed-dose tablets and pioglitazone and metformin given separately has been established in terms of absorption and bioavailability parameters in two studies in which healthy volunteers were treated with a fixed-dose combination of pioglitazone and metformin at two dose strengths, $15 \mathrm{mg} / 500 \mathrm{mg}$ and $15 \mathrm{mg} / 850 \mathrm{mg}$, or with the coadministration of each drug separately (Karim et al. 2007a,b). Moreover, food had no effect on the bioavailability of the fixed-dose combination compared with the separate drugs.

\section{Glycemic control}

To date, the efficacy of the combined treatment with pioglitazone and metformin may be assumed from the results of combination clinical trials, since there are no data on glycemic control provided by a fixed-dose combination in clinical trials (Seufert 2006). Several randomized placebo-controlled or active comparator trials have evaluated the efficacy of treatment with pioglitazone and metformin on glycemic control. In a 16-week trial, combination therapy with pioglitazone and metformin significantly improved $\mathrm{HbA}_{1 \mathrm{c}}$ (difference in mean change between groups $-0.83 \% ; P \leq 0.05$ ) and fasting plasma glucose (FPG; difference in mean change between groups $-37.7 \mathrm{mg} / \mathrm{dL} ; P \leq 0.05)$ in diabetic patients inadequately controlled with metformin (Einhorn et al. 2000). A significant difference from baseline was observed at week 4 for both parameters. The positive effect of pioglitazone was sustained during the 72-week open-label extension phase of this study. Patients treated with pioglitazone plus metformin in the open-label phase had mean changes from baseline of $-1.36 \%$ in $\mathrm{HbA}_{1 \mathrm{c}}$ and $-63.0 \mathrm{mg} / \mathrm{dL}$ in FPG. The placebo plus metformin group showed a small mean increase in fasting C-peptide from baseline $(+0.1 \mathrm{ng} / \mathrm{mL})$ while in the pioglitazone plus metformin group a small decrease was registered $(-0.1 \mathrm{ng} / \mathrm{mL})$, the difference becoming significant at week $16(P \leq 0.05)$ (Einhorn et al. 2000).

Mounting evidence shows that pioglitazone in association with metformin improves glycemic control to a similar extent as the combination of rosiglitazone and metformin. In a 12-month randomized clinical trial that directly compared the combination of pioglitazone and metformin with rosiglitazone and metformin, pioglitazone combined with metformin induced a significant improvement in glycemic control $(P<0.05)$ after 9 months of treatment, although there was no significant difference between groups at the end of the study. Reduction in FPG and postprandial blood glucose were also similar between groups $(P<0.05)$. Another primary endpoint of this comparator study was the effect of the two combinations on lipid profile, which is discussed below (Derosa et al. 2007a).

The efficacy of pioglitazone and metformin combination treatment has been compared with that of gliclazide and glibenclamide. In comparison with gliclazide added to metformin, pioglitazone addon therapy induced a similar reduction of $\mathrm{HbA}_{1 \mathrm{c}}(-0.99 \%$ vs $-1.01 \%$ ), while improvement of FPG was significantly greater in patients receiving pioglitazone plus metformin $(-2.1$ vs $+1.6 \mathrm{mmol} / \mathrm{L})(P<0.001)$ (Charbonell et al. 2005). Improved glycemic control was effectively sustained by pioglitazone and metformin for the 52-week extension of this study, with a statistically significant difference between groups in a 
per-protocol analysis of patients who had been treated for $\geq 18$ months $(-1.07 \%$ vs $-0.76 \%$ ) (Matthews et al. 2005$)$. In a 26-week study, no statistically significant differences were found between pioglitazone plus metformin and pioglitazone plus glimepiride with regard to both FPG and $\mathrm{HbA}_{1 \mathrm{c}}(-1.23 \%$ and $-1.30 \%$, respectively) (Umpierrez et al 2006).

Concerning studies comparing pioglitazone in association with either metformin or glibenclamide, both combinations were equally effective in terms of reduction of $\mathrm{HbA}_{1 \mathrm{c}}$ in two long-term prospective trials. In a 3-year study, reduction in $\mathrm{HbA}_{1 \mathrm{c}}$ at 72 weeks was greater in the pioglitazone and metformin group $(P<0.001)$; at 156 weeks, changes in $\mathrm{HbA}_{1 \mathrm{c}}$ were $-2.3 \%$ and $+1.8 \%$, respectively, with pioglitazone plus metformin and plus glibenclamide (Spanheimer et al. 2006). At the end of a second study, which lasted for 96 weeks, the reduction in $\mathrm{HbA}_{1 \mathrm{c}}$ was significantly greater in the pioglitazone plus metformin group $(-0.85 \%$ vs $-0.61 \% ; P=0.025)$ (Oerter et al. 2006). In one observational study, significantly fewer patients required a shift to insulin treatment if they received pioglitazone plus metformin $(P<0.001)$ (Deeks \& Scott 2006; Mirkolf et al. 2006).

As regards insulin sensitivity, one study was considered in our evaluation of the literature. Pioglitazone plus metformin has been evaluated in a clinical trial in which the primary outcome was the comparison with gliclazide and metformin on afterload glycemia and a composite index of insulin sensitivity (CISI) (Ceriello et al. 2005). Results of this study show that treatment with pioglitazone plus metformin reduced fasting insulinemia while it was increased by the combination of pioglitazone and gliclazide. $\mathrm{HbA}_{1 \mathrm{c}}$ was lowered by $1 \%$ and $\mathrm{FPG}$ was reduced by $1.53 \mathrm{mmol} / \mathrm{L}$ to $1.29 \mathrm{mmol} / \mathrm{L}$ without significant differences between groups (Ceriello et al. 2005).

\section{Cardiovascular events}

Recently, a large clinical trial, PROactive, demonstrated the efficacy of pioglitazone in reducing cardiovascular events (Dormandy et al. 2005). The study showed that treatment with pioglitazone is effective in improving a series of cardiovascular risk factors, thus resulting in a general reduction of cardiovascular morbidity and mortality; tolerability of pioglitazone at different dosages (from $15 \mathrm{mg} /$ day to $45 \mathrm{mg} /$ day) was also evaluated. All patients enrolled presented with macrovascular disease such as recent ( $<6$ months) acute myocardial infarction (AMI), acute coronary syndrome, revascularization, stroke, or severe peripheral arterial disease. The composite primary endpoint was the time required for the presentation of a second cardiovascular event after randomization: death from all causes, nonfatal myocardial infarction (including silent AMI), stroke, acute coronary syndrome, coronary or peripheral revascularization, and amputation. Secondary endpoints were the time preceding the first all-cause death, AMI (excluding silent AMI), and stroke (main secondary endpoint). The primary endpoint was reduced by $10 \%$ by pioglitazone titrated from 15 to $45 \mathrm{mg}$ [pioglitazone 514/2605 events vs placebo 572/2633; hazard ratio (HR) 0.90; $95 \% \mathrm{Cl}: 0.80$, $1.02 ; P=0.095]$. When the primary endpoint includes only clinical objectives, the reduction was significant in the pioglitazone group versus placebo (16\%; $P=0.027)$. Inclusion of revascularization events in the primary composite endpoint reduces the significance of the results, since revascularization depends on the likelihood of different study centres to perform this surgical intervention.

PROactive thus demonstrated a reduction of AMI, stroke, acute coronary syndrome, and cardiac revascularization in patients treated with pioglitazone compared with placebo. Treatment with pioglitazone showed a good tolerability profile in a large number of patients. The more frequently reported adverse events were infectious respiratory disease, headache, and muscle pain.

Level 2 evidence has shown the clinical efficacy of metformin in reducing cardiovascular events in overweight patients with type 2 diabetes. Patients treated with metformin had a risk reduction of $32 \%$ for any endpoint related to diabetes, $42 \%$ for diabetesrelated death $(P=0.017)$, and $36 \%$ for all mortality $(P=0.011)$. Moreover, metformin did not induce weight gain and hypoglycemia (UKPDS 1998).

\section{Reduced cardiovascular risk}

Level 2 evidence has shown that combination therapy with pioglitazone and metformin is effective in reducing a number of cardiovascular risk factors. Most of the results obtained in this context show that the reduction of some conventional (Reaven 1988) and nonconventional cardiovascular risk factors are independent of the achievement of recommended glycemic control. Recent findings about the potential increase and/or reduction of cardiovascular disease with TZDs are discussed below. Here we analyze the data on treatment with pioglitazone and pioglitazone plus metformin on cardiovascular risk factors.

Pioglitazone in association with metformin produced a significant reduction of plasminogen activator inhibitor 1 (PAI-1) in patients with type 2 diabetes and metabolic syndrome $(P<0.05)$ (Derosa et al. 2006a). When compared with rosiglitazone/metformin, reduction in PAl-1 levels is similar after 1 year of combination treatment. Metformin is known to reduce triglycerides, PAI-1, factor VII, and C-reactive protein (CRP) (Després 2003). Few studies are available investigating the antithrombotic effects of pioglitazone or rosiglitazone in combination with metformin, and no studies exist that directly compare the two different combinations of pioglitazone/metformin and rosiglitazone/ metformin on $\mathrm{PAl}-1$ reduction.

Recently, the effects of pioglitazone in association with metformin on other important nonconventional cardiovascular risk factors have been studied. Lipoprotein-a [Lp-(a)] levels have been shown to be related to an increased risk of atherosclerosis. Lp-(a) levels are augmented in diabetic patients and are involved in the development of some typical complications of diabetes such as retinopathy and percutaneous transluminal angioplasty restenosis (Maca et al. 2002). Diabetic patients also often present with increased levels of homocysteine (Hcy), or hyperhomocysteinemia, which appears to be an independent risk 
factor for atherothrombotic events (Herrmann \& Knapp 2002). In a 12-month study, pioglitazone and metformin were compared with rosiglitazone and metformin in order to evaluate the effects of these combinations on reduction of Lp-(a) and Hcy in type 2 diabetic patients. At the end of the study no differences were observed between the two combinations on metabolic improvement and reduction in Hcy levels, while reduction in Lp-(a) was observed only in the pioglitazone/metformin group (Derosa et al. 2006b). When the effects of combinations of glimepiride with pioglitazone and glimepiride with rosiglitazone on homocysteinemia and Lp-(a) were evaluated, both combinations improved Hcy levels, but only the pioglitazone combination was associated with significant reduction in Lp-(a) levels (Derosa et al. 2006c). In both studies pioglitazone was administered at the lowest dosage $(15 \mathrm{mg})$ and results on Lp-(a) levels were obtained after 1 year. No long-term clinical studies have evaluated the effects of pioglitazone and metformin on prothrombotic states in a large cohort of diabetic patients, and no data are available on the maintenance of a more favorable coagulative profile after administration of pioglitazone/metformin fixed-dose combination.

\section{Hypertension}

Based on level 1 evidence, hypertension is a well-recognized high-risk condition in diabetic subjects and even more restrictive recommendations have been published for the optimal control of blood pressure values in diabetic patients (ADA 2007; Fuster et al. 2006). Strong evidence suggests that TZDs positively affect blood pressure values in diabetic patients. A recent meta analysis of 32 clinical trials showed that both pioglitazone and rosiglitazone reduce SBP by $4.7 \mathrm{mmHg}$ and DBP by $43.8 \mathrm{mmHg}$. Compared with placebo, TZD treatment resulted in a reduction of $3.47 \mathrm{mmHg}$ and $1.84 \mathrm{mmHg}$ for SBP and DBP, respectively (Qayyum \& Adomaytyte 2006). A combination of pioglitazone and metformin is effective in reducing SBP and DBP in type 2 diabetic patients who have lost the physiologic nocturnal reduction of both SBP and DBP (so-called "nondippers"). This effect appears to be independent of the amelioration of metabolic parameters (Negro et al. 2004). Similar effects have been observed with rosiglitazone; in association with metformin, rosiglitazone reduces both SBP and DBP and restores physiologic decreases in blood pressure during the night in nondipping diabetic patients (Negro et al. 2005). Twelve months' comparison between pioglitazone or rosiglitazone in association with metformin shows that both combinations result in significant improvements in SBP and DBP $(P<0.05)$ and a comparable amelioration of metabolic parameters and proinflammatory state (Derosa et al. 2007b). The combination of TZD and metformin is associated with a slight but significant improvement in the longterm blood pressure control of these patients and with an improvement in the antiinflammatory state, both of which are related to a similar reduction in insulin resistance (Derosa et al. 2007b).

All these results are in agreement with the level 1 evidence that TZDs are effective in reducing blood pressure and modifying cardiovascular risk factors, and particularly macrovascular complications, in association with metformin or SUs (Meriden 2004; Granberry \& Fonseca 2005).

Both pioglitazone and rosiglitazone have been shown to improve microalbuminuria. The results obtained in randomized controlled trials show that the beneficial effects of TZDs on microalbuminuria are independent of the improvement in metabolic parameters. Compared to metformin, pioglitazone has been shown to reduce microalbuminuria in diabetic patients, alone or in combination with a SU. In a large clinical trial (QUARTET) the main outcome in diabetic patients treated with pioglitazone in association with a SU versus metformin combination with a $\mathrm{SU}$ was the reduction of $\mathrm{HbA}_{1 \mathrm{c}}$ by $1.2 \%$ for both treatments; a secondary endpoint was the reduction in microalbuminuria after combination treatment. Pioglitazone plus a SU resulted in a significant reduction in albumin/creatinine excretion rate $(-15 \%)$ compared with metformin, which induced a worsening (Hanefeld et al. 2004; Schernthaner et al. 2004).

TZDs, compared with other antidiabetic drugs such as SUs and metformin, have unique effects on microalbuminuria. Other data show that the urinary albumin/creatinine ratio is reduced by the pioglitazone/metformin combination compared with gliclazide/metformin $(P<0.05)$ (Matthews et al. 2005). Some data reported in level 1 evidence show that the urinary albumin/creatinine ratio was also reduced in a study of pioglitazone add-on to metformin (-10\%), compared to gliclazide add-on with $(6 \% ; P<0.05)($ Erdmann 2006).

\section{Lipid abnormalities}

A positive effect of TZDs on lipid profile and lipoprotein subfractions has been recently observed in a number of studies. The differential effects of rosiglitazone and pioglitazone on lipid profile have been extensively studied (van Wijk et al. 2003), and available data suggest a substantial superiority of pioglitazone compared with rosiglitazone in improving lipid abnormalities. Pioglitazone and rosiglitazone have significantly different effects on plasma lipids independent of glycemic control or concomitant lipid-lowering or other antihyperglycemic therapy. Compared with rosiglitazone, pioglitazone is associated with improvements in triglycerides, high-density lipoprotein cholesterol (HDL-C), LDL particle concentration, and LDL particle size (Goldberg et al. 2005).

Data are available on the effect of TZDs in combination with metformin and SUs on lipids. In a recent clinical trial, glimepiride plus pioglitazone significantly improved lipid and lipoprotein variables, whereas the combination of glimepiride plus rosiglitazone appears to have no clinically significant effect on lipid metabolism in patients with type 2 diabetes and metabolic syndrome. Treatment with pioglitazone and glimepiride significantly lowered total cholesterol (-11\%), LDL-C (-12\%), HDL-C (15\%), and apolipoprotein B (Apo-B) $(-10.6 \%) \quad(P=0.05)$, while treatment with rosiglitazone and glimepiride increased total cholesterol (+14.9\%), LDL-C $(+16.5 \%)$, triglycerides $(+17.9 \%)$, and Apo-B $(+10.3 \%)(P<0.05)$ (Derosa et al. 2004). 
The effects of rosiglitazone and pioglitazone on lipids have been compared in 127 patients with type 2 diabetes who had been previously treated with troglitazone. After 4 months of treatment both groups had a significant increase in BMI $(P<0.01)$. No significant differences were observed in lipid profiles in the group treated with rosiglitazone, while in pioglitazone recipients a significant improvement was obtained in total cholesterol levels $(P<0.01)$ after adjustment for demographic profile and treatment with statins (Khan et al. 2002). In a long-term clinical trial the combination of metformin and pioglitazone was more effective than both gliclazide plus pioglitazone, and gliclazide plus metformin in improving lipid profiles $(P<0.001)$. An analysis of long-term studies over 2 years revealed that the effectiveness of combination treatment with pioglitazone and metformin was superior to pioglitazone plus gliclazide and metformin plus gliclazide. In particular, combination treatment with pioglitazone and metformin induced a statistically significant reduction in triglycerides that was sustained over time, with decreases of $16-18 \%$ at 1 year and $17-23 \%$ at 2 years $(P<0.01)$, and an increase in HDL levels, both sustained to the end of the study. A small but significantly greater reduction in LDL-C was obtained in the group treated with gliclazide and metformin versus both pioglitazone and metformin and pioglitazone and gliclazide. Mean fasting levels of nonesterified fatty acids were decreased more in the pioglitazone and metformin group than in the gliclazide and metformin group $(P=0.046)$ (Betteridge \& Verges 2005).

Data from another large clinical trial show that addition of pioglitazone to metformin and SUs alone or in combination was more effective than adding rosiglitazone in reducing triglycerides levels $(P<0.001)$ and increasing HDL levels $(P<0.001)$. The results were consistent among all the treatment subgroups analyzed in the study (Peters Harmel et al. 2004). Both in monotherapy and add-on therapy to metformin and SUs, pioglitazone has been shown to improve LDL particle size and lead to an antiatherogenic lipid profile compared with other add-on therapy such as metformin and SUs (Khan et al. 2004).

No large long-term clinical trials have been found that directly compare the effects of combining metformin and pioglitazone with other combination therapy on lipid profile and particle size and subclass distribution of lipoproteins. Nevertheless, in a direct comparison between pioglitazone plus metformin and rosiglitazone plus metformin, a significant lipid profile improvement was present in the pioglitazone group at 12 months compared with the baseline values, and these variations were significantly different between groups (Derosa et al. 2006b). Pioglitazone and metformin combination treatment has also shown positive effects on LDL subfractions (Lawrence et al. 2004). Pioglitazone and metformin combination treatment reduced LDL particle size and apolipoprotein levels [Apo-Al, Apo-Al/All-containing HDL, and Lp-(a)] and increased Apo-B (Perez et al. 2004).

Increasing evidence indicates an endocrine role of adipose tissue, producing a series of endocrinologically active molecules, adipocytokines. Diabetic patients usually have low levels of adiponectin, which is inversely related to insulin sensitivity. In a 12-week study, pioglitazone monotherapy increased adiponectin levels in type 2 diabetic patients compared with metformin, while leptin levels (reduced in diabetic patients at baseline) appeared unaffected by pioglitazone monotherapy and improved by metformin (Sharma et al. 2006). Fixed-dose combination studies are not available investigating lipid effects.

\section{Safety and tolerability}

According to level 2 evidence, oral administration of pioglitazone (30-45 mg) plus metformin therapy was generally well tolerated in long-term clinical trials (Einhorn et al. 2000; Matthews et al. 2005). Data come primarily from descriptive analyses and are partially derived from the manufacturer's prescribing information (Anon. 2007).

Combination treatment with pioglitazone and metformin presents a tolerability profile comparable to metformin monotherapy, the common side effects of which are principally nausea, diarrhea, and gastrointestinal discomfort. The safety and tolerability of pioglitazone in combination with metformin was compared with rosiglitazone plus metformin in a 12-month study. With both treatments no statistically significant change was detected compared with baseline values (Derosa et al. 2006b). Clinical trials report a low incidence of edema (peripheral and combined) and headache (Deeks \& Scott 2006). Tolerability of the two dosages of pioglitazone (30 and $45 \mathrm{mg}$ ) was similar, with a small difference in limb edema according to a 52-week clinical trial (Matthews et al. 2005).

\section{Cardiac events}

Cardiovascular risk has recently become the key issue in TZD therapy, and cardiovascular risk of a fixed-dose combination cannot be analyzed without including the recent findings in the literature. A recent meta analysis of 42 randomized control trials involving 28443 patients treated with rosiglitazone showed an increase in the risk of $\mathrm{AMI}$ compared with controls (odds ratio 1.43; 95\% Cl 1.03, 1.98; $P=0.03$ ) (Nissen \& Wolski 2007). A subsequent meta analysis included randomized clinical trials with a specified intention to evaluate cardiovascular events in diabetic and nondiabetic populations (prediabetic). The studies included had almost a 12-month follow-up period and provided numerical data of cardiovascular side effects. Results of this analysis showed that rosiglitazone increases the risk of AMI [relative risk (RR) $1.42 \%$; $95 \% \mathrm{Cl} 1.06,1.91 ; P=0.02]$ and congestive heart failure (RR 2.09; 95\% Cl 1.52, 2.88; $P<0.001)$ without raising the risk of cardiovascular mortality (RR $0.90 ; 95 \% \mathrm{Cl} 0.63,1.26$; $P=0.53$ ) (Nissen et al. 2007). The limitations of collecting data from the studies included in the analysis and the limitations of a meta analysis in itself are analyzed in the paper. After having considered these data, the rosiglitazone label was modified by the Food and Drug Administration in August 2007 (Singh et al. 2007).

Data from the PROactive trial, as explained above, revealed a favorable impact of pioglitazone on cardiovascular risk. In particular, long-term treatment with pioglitazone seems to reduce the risk of AMI. However, the issues raised by the meta analysis of Nissen et al. (2007) have made it necessary to better 
understand the potential of an increase in AMI in patients treated with pioglitazone. In another recent meta analysis, the effect of pioglitazone on ischemic and cardiovascular events has been investigated (Lincoff et al. 2007). The studies included $(n=19)$ in this analysis had to be randomized clinical trials, double-blind and controlled with placebo or active comparator. The primary outcome measure was a composite of death, AMI, or stroke, and secondary endpoint measures included the incidence of serious heart failure. Death, AMI, or stroke occurred in $4.4 \%$ of patients receiving pioglitazone versus $5.7 \%$ patients receiving control drugs (HR 0.82; 95\% Cl 0.72, 0.94; $P=0.005)$, with a similar magnitude in reduction of each individual component of the primary endpoint (HR range 0.80-0.92), while serious heart failure was registered in $2.3 \%$ of the patients treated with pioglitazone versus $1.8 \%$ of patients receiving control drug therapy (HR 1.41; $95 \% \mathrm{Cl} 1.14,1.76 ; P=0.002)$.

In summary, the evidence indicates that pioglitazone is associated with a reduced risk of death, AMI, or stroke among a diverse population of patients with type 2 diabetes. The drug was associated with an increased risk of heart failure, but without an increase in resultant mortality.

\section{Edema}

Edema represents one of the most common and undesirable side effects of treatment with a TZD, and appears to be more common when TZDs are used in combination therapy. In the study by Matthews et al. (2005), the incidence of edema in patients treated with pioglitazone and metformin was $6.3 \%$, compared with $2.2 \%$ in patients treated with gliclazide and metformin. One patient discontinued treatment because of edema, while pulmonary edema developed in two patients, which was considered to be related to drug therapy in one patient and was accompanied by AMI (Matthews et al. 2005). A number of studies report an increased risk of edema in patients treated with pioglitazone, and in combination studies (not fixed-dose studies) this event has also been reported. In the study by Einhorn et al. (2000) peripheral edema and central edema occurred in $5.9 \%$ of the pioglitazone plus metformin group, but it was described as mild to moderate. No studies with the fixed-dose combination of pioglitazone/ metformin are available that directly evaluate the increase of edema with this formulation.

Fluid retention with TZDs has been associated with different mechanisms and may be considered a multifactorial process. Level 1 evidence is available regarding these different mechanisms, and it may also be considered in the context of recent controversies about heart failure with TZDs (see Place in therapy). The evidence indicates that pioglitazone and rosiglitazone induce edema more in combination therapy than as monotherapy, and in particular when administered with insulin or SUs (Nesto et al. 2004). Excluding insulin combination therapy, there is no apparent difference between the different combinations with pioglitazone or rosiglitazone and no headto-head comparisons have been performed or reported to investigate this effect.

\section{Aminotransferase increase}

Increase of alanine aminotransferase (ALT) levels $(\geq 3$ times the upper level) was limited in clinical trials (Anon. 2007). In a 3-year randomized double-blind safety study (Spanheimer et al. 2006) the incidence of ALT elevation $\geq 3$ times the upper limit during treatment with pioglitazone and metformin was comparable to that with glibenclamide and metformin. In the same study, the incidence of ALT and gamma-glutamyl-transpeptidase $>1.5$ the upper limit and/or baseline was significantly more frequent with glibenclamide than with pioglitazone/metformin. Aspartate aminotransferase (AST) elevation was more frequent with pioglitazone but the mean change of AST and ALT concentrations were lower in the pioglitazone group (Spanheimer et al. 2006).

\section{Hypoglycemia}

As reported in clinical trials, hypoglycemia was less frequent with pioglitazone in association with metformin than with the combination of SUs and metformin. These data have been confirmed in short- and long-term clinical trials that have compared pioglitazone in association metformin with the different SUs glimepiride (Umpierrez et al. 2006), gliclazide (Charbonell et al. 2005), and glibenclamide (Perez et al. 2006) when given with metformin.

\section{Lactic acidosis}

Lactic acidosis occurs primarily in patients treated with metformin, particularly those with renal impairment, hypoxia, heart failure, and other risk factors for acidosis. According to data from clinical trials there were no reported cases of lactic acidosis in more than $\mathbf{2 0} 000$ patient-years with pioglitazone and metformin combination treatment (Anon. 2007). The reported incidence of lactic acidosis in patients receiving metformin is very low (0.03 cases $/ 1000$ patient-years). Reported cases occurred in patients with renal insufficiency, advanced age, heart failure, excessive alcohol intake, intravascular radiocontrast injection, and respiratory disease. No evidence exists regarding the risk of lactic acidosis with pioglitazone or fixed-dose pioglitazone and metformin add-on therapy (Anon. 2007).

\section{Increase in bodyweight}

A moderate bodyweight gain is well known in patients treated with TZDs, due to the stimulation of preadipocyte differentiation. In a 12-month comparative study between pioglitazone and rosiglitazone associated with metformin, no BMI changes were observed in either group (Derosa et al. 2007a). Combination with pioglitazone gives rise to a small increase in bodyweight when compared with metformin alone, which slightly decreases bodyweight (+0.95 kg vs $-1.36 \mathrm{~kg}$ after 16 weeks) (Einhorn et al. 2000). In other studies, combination treatment of pioglitazone and metformin was associated with weight gain, to a similar degree to that observed with metformin and gliclazide $(+1.5 \mathrm{~kg}$ vs $+1.4 \mathrm{~kg}$ compared with gliclazide alone after 52 weeks) (Matthews et al. 2005) and glimepiride (+1.74 kg vs $+1.85 \mathrm{~kg}$ after 28 weeks) (Umpierrez et al. 2006). 


\section{Economic evidence}

No economic studies are available for the fixed-dose pioglitazone and metformin formulation; a health technology assessment on pioglitazone and rosiglitazone pubished in 2004 also reported no published economic studies on either drug (Czoski-Murray et al. 2004). Direct head-to-head comparisons of the glitazones in combination with metformin or SUs would be helpful.

\section{Dosage, administration, and formulations}

Fixed-dose tablets of pioglitazone/metformin are approved in the US (15 mg/500 mg and $15 \mathrm{mg} / 850 \mathrm{mg}$ ) and EU (15 mg/850 mg) for the treatment of patients with type 2 diabetes who fail to achieve glycemic control with monotherapy, and monotherapy with metformin, respectively. In the US, in inadequately controlled glycemia with pioglitazone or metformin alone, and in patients currently receiving pioglitazone plus metformin, the recommended dosage of the pioglitazone/metformin combination is $15 \mathrm{mg} / 500 \mathrm{mg}$ or $15 \mathrm{mg} / 850 \mathrm{mg}$ once or twice daily. The maximum recommended dosage is $45 \mathrm{mg} / 2550 \mathrm{mg}$ per day. In the EU, $15 \mathrm{mg} / 850 \mathrm{mg}$ twice daily is the recommended dosage in patients in whom glycemia is inadequately controlled with metformin alone.

\section{Place in therapy}

As discussed in this review, pioglitazone and metformin coadministration improves glycemic control, insulin sensitivity, and lipid profiles, which remain the cardinal points of diabetes care. The different mechanism of action of two insulin-sensitizing agents leads to the optimized control of the different pathways that lead to insulin resistance, inflammation, and atherosclerosis. Despite the lack of long-term clinical trials of the fixed-dose combination of pioglitazone and metformin in the management of cardiovascular risk factors, the evidence of a possible effect of pioglitazone on proinflammatory markers, adipocytokines, and procoagulative state supports its use in the treatment of metabolic syndrome. On the other hand, recent data from meta analyses of rosiglitazone and pioglitazone have to be considered before discussing the possible role of a fixed-dose combination of pioglitazone and metformin in prevention of cardiovascular risk. The major limits observed in meta analyses of rosiglitazone and pioglitazone are the unfortunate lack of studies intended to assess cardiovascular outcomes in patients treated with TZDs (Lincoff et al. 2007; Singh et al. 2007). No studies are available where the primary outcome is cardiovascular mortality with the fixed association of metformin plus a TZD. Large clinical trials investigating cardiovascular outcomes in patients treated with pioglitazone monotherapy are required, as are studies on the comparison between pioglitazone with other older drugs such as metformin and SUs. A retrospective analysis conducted in patients treated with antidiabetic drugs other than TZDs (metformin, SUs, or insulin) as monotherapy or combination therapy demonstrated that the incidence of heart failure among patients with type 2 diabetes is more frequent with any drug therapy, with little difference between treatments. The risk of $\mathrm{AMI}$ seems to be slightly higher during the first year, with risk of heart failure decreasing thereafter to become comparable to that in patients treated with diet and physical activity. Regarding pioglitazone either alone or in combination with metformin, no studies are available that can explain such a relationship.

In this brief discussion many unsolved questions emerge relating to the real risk (rosiglitazone) and/or benefit (pioglitazone) TZDs could bring in the prevention of cardiovascular events. For example, how many studies are reliable enough to make conclusions about cardiovascular outcomes? And are the studies comparable? There is a lack of evidence in this regard on newer formulations such as pioglitazone plus metformin and rosiglitazone plus metformin in fixed-dose combinations, but there is evidence that these combinations improve glycemic control in type 2 diabetes. There are two issues that need to be considered: the direct and/or indirect mechanisms that link hyperglycemia itself and insulin resistance with coronary damage and atherosclerosis development are still partially unknown, and the interaction between pioglitazone and metformin with the pathophysiological steps that lead to atherosclerosis in some metabolic diseases such as diabetes.

Despite these issues, our opinion is that glycemic control remains the objective of treatment, and strong evidence exists for the beneficial effect of a fixed-dose combination. Obesity may be managed as one of the major steps to early atherosclerosis and the assessment of fat tissue should be one of the outcomes for clinicians.

Diabetic patients are often treated with multiple drugs, and may require a more practical and convenient therapeutic regimen, which can be provided by a fixed-dose combination. Side effects do not appear to be increased when a fixed dose is compared with monotherapy or other combination therapy, when drugs are administered according to manufacturers' advice. More studies are needed on the potential economic impact of fixed-dose combination treatment with pioglitazone and metformin, and large-scale, long-term clinical trials will provide clear evidence of the potential preventive effects on macro- and microvascular complications of type 2 diabetes.

\section{Acknowledgments}

The authors declare that they have no conflict of interest.

\section{References}

ADA (American Diabetes Association). Standards of medical care in diabetes 2007. Diabetes Care. 2007;30(Suppl. 1):S4-S41.

Ahmann AJ, Riddle MC. Current oral agents for type 2 diabetes. Many options but which to choose when? Postgrad Med. 2002;111:32-46.

Anon. Actoplus prescribing information. Deerfield, IL: Takeda Pharmaceuticals America, Inc.; 2007. Available at:

http://www.actos.com/actos/prescribinginfo.aspx (accessed August 2007).

Betteridge DJ, Verges B. Long-term effects on lipids and lipoproteins of pioglitazone versus gliclazide addition to metformin and pioglitazone versus metformin addition to sulphonylurea in the treatment of type 2 diabetes. Diabetologia. 2005;48:2477-2481.

Bays HE, Bazata DD, Clark NG, et al. Prevalence of self-reported diagnosis of diabetes mellitus and associated risk factors in a national survey in the US population: SHIELD (Study to Help Improve Early evaluation and management of risk factors Leading to Diabetes). BMC Public Health. 2007;7:277-303. 
Bolen S, Feldman L, Vassy J, et al. Systematic review: comparative effectiveness and safety of oral medications for type 2 diabetes mellitus. Ann Intern Med. 2007;147:386-399.

Ceriello A, Johns D, Widel M, et al. Comparison of the effect of pioglitazone with metformin or sulphonylurea (monotherapy and combination therapy) on post-load glycemia and composite insulin sensitivity index during an oral glucose tolerance test in patients with type 2 diabetes. Diabetes Care. 2005;28:266-272.

Charbonell BH, Schernthaner G, Brunetti P, et al. Long-term efficacy and tolerability of add-on pioglitazone therapy to failing monotherapy compared with addition of gliclazide or metformin in patients with type 2 diabetes. Diabetologia. 2005;48:1093-1104.

Czoski-Murray $\mathrm{C}$, Warren $\mathrm{E}$, Chilcott $\mathrm{J}$, et al. Clinical effectiveness and costeffectiveness of pioglitazone and rosiglitazone in the treatment of type 2 diabetes: a systematic review and economic evaluation. Health Technol Assess. 2004;8:iii,ix-x, 1-91.

Deeks ED, Scott LJ. Pioglitazone/metformin. Drugs. 2006;66:1863-1877.

Derosa G, Cicero AF, Gaddi A, et al. Metabolic effects of pioglitazone and rosiglitazone in patients with diabetes and metabolic syndrome treated with glimepiride: a twelve-month, multicenter, double-blind, randomized, controlled, parallel-group trial. Clin Ther. 2004;26:744-754.

Derosa G, D'Angelo A, Ragonesi PD, et al. Effects of rosiglitazone and pioglitazone combined with metformin on the prothrombotic state of patients with type 2 diabetes mellitus and metabolic syndrome. J Int Med Res. 2006a;34:545-555.

Derosa G, D’Angelo A, Ragonesi PD, et al. Metformin-pioglitazone and metformin-rosiglitazone effects on non-conventional cardiovascular risk factors plasma level in type 2 diabetic patients with metabolic syndrome. $J$ Clin Pharm Ther. 2006b;31:375-383.

Derosa G, Cicero AFG, D'Angelo A, et al. Effects of 1 year of treatment with pioglitazone or rosiglitazone added to glimepiride on lipoprotein (a) and homocysteine concentrations in patients with type 2 diabetes mellitus and metabolic syndrome: a multicenter, randomized, double-blind, controlled clinical trial. Clin Ther. 2006c;28:679-688. (erratum: Clin Ther. 2006;28:1483.)

Derosa G, D'Angelo A, Ragonesi PD, et al. Metabolic effects of pioglitazone and rosiglitazone in patients with diabetes and metabolic syndrome treated with metformin. Int Med J. 2007a;37:79-86.

Derosa G, Fogari E, Cicero AF, et al. Blood pressure control and inflammatory markers in type 2 diabetic patients treated with pioglitazone or rosiglitazone and metformin. Hypertens Res. 2007b;30:387-394.

Després JP. Potential contribution of metformin in the management of cardiovascular disease risk in patients with abdominal obesity, the metabolic syndrome and type 2 diabetes. Diabetes Metab. 2003;29:6S53-6S61.

Dormandy JA, Charbonnel B, Eckland DJ, et al. Secondary prevention of macrovascular events in patients with type 2 diabetes in the PROactive Study (PROspective pioglitAzone Clinical Trial In macroVascular Events): a randomised controlled trial. Lancet. 2005;366:1279-1286.

Einhorn D, Rendell M, Rosenzweig J, et al. Pioglitazone hydrochloride in combination with metformin in the treatment of type 2 diabetes mellitus: a randomized, placebo-controlled study. The Pioglitazone 027 Study Group. Clin Ther. 2000;22:1395-1409.

Erdmann E. Microalbuminuria as a marker of cardiovascular risk in patients with type 2 diabetes. Int J Cardiol. 2006;107:147-153.

Gilmer TP, Roze S, Valentine WJ, et al. Cost-effectiveness of diabetes case management for low-income populations. Health Serv Res. 2007;42:1943-1959.

Goldberg RB, Kendall DM, Deeg MA, et al. GLAI Study Investigators. A comparison of lipid and glycemic effects of pioglitazone and rosiglitazone in patients with type 2 diabetes and dyslipidaemia. Diabetes Care. 2005;28:1547-1554.

Granberry MC, Fonseca VA. Cardiovascular risk factors associated with insulin resistance: effects of oral antidiabetic agents. Am J Cardiovasc Drugs. 2005;5:201-209.

Haffner SM. Abdominal adiposity and cardiometabolic risk: do we have all the answers?. Am J Med. 2007;120(Suppl. 1):s10-s16.
Hanefeld N, Brunetti P, Schernthaner GH, et al. One-year glycemic control with a sulphonylurea plus piolgitazone versus a sulphonylurea plus metformin in patients with type 2 diabetes. Diabetes Care. 2004;27:141-147.

Herrmann W, Knapp JP. Hyperhomocysteinemia: a new risk factor for degenerative disease. Clin Lab. 2002;48:471-481.

IDF (International Diabetes Federation). Diabetes Atlas. 3rd ed. Brussels: International Diabetes Federation; 2006. Available at http://www.eatlas.idf.org (accessed December 5, 2007).

Lincoff AM, Wolski K, Nicholls SJ, Nissen SE. Pioglitazone and the risk of cardiovascular events in patients with type 2 diabetes mellitus. A meta-analysis of randomized trials. JAMA. 2007;298:1180-1188.

Jain R, Chawrai S. Advancements in the anti-diabetes chemotherapeutic based on amino acids, peptids and peptidomimetics. Mini Rev Med Chem. 2005;5:469-477.

Karim A, Slater M, Bradford D, Schwartz L, Laurent A. Oral antidiabetic drugs: effect of food on absorption of pioglitazone and metformin from a fixed-dose combination tablet. J Clin Pharmacol. 2007a;47:48-55.

Karim A, Slater M, Bradford D, et al. Oral antidiabetic drugs: bioavailability assessment of fixed-dose combination tablets of pioglitazone and metformin. Effect on body weight, gender and race on systemic exposures of each drug. J Clin Pharmacol. 2007b;47:37-47.

Khan MA, St Peter JV, Xue JL. A prospective, randomized comparison of the metabolic effects of pioglitazone or rosiglitazone in patients with type 2 diabetes who were previously treated with troglitazone. Diabetes Care. 2002;25:708-711.

Khan M, Xu Y, Edwards G, et al. Effects of pioglitazone on the components of diabetic dyslipidemia: results of double-blind multicenter randomised studies. Int J Clin Pract. 2004;58:907-912.

Krentz AJ, Bailey CJ. Oral antidiabetic agents. Drugs. 2005;65:385-411.

Kyvik KO, Green A, Beck-Nielsen H. Concordance rates of insulin dependent diabetes mellitus : a population race study of young Danish twins. $\underline{B M J}$. 1995;311:913-917.

Lawrence JM, Reid J, Taylor GJ, et al. Favorable effects of pioglitazone and metformin compared with gliclazide on lipoprotein subfractions in overweight patients with early type 2 diabetes. Diabetes Care. 2004;27:41-46.

Maca TH, Ahmadi R, Dergfler K, et al. Influence of lipoprotein (a) on restenosis after femoro-popliteal percutaneous transluminal angioplasty in type 2 diabetic patients. Diab Med. 2002;19:300-306.

Mann Jl. Diet and risk of coronary heart disease and type 2 diabetes. Lancet. 2002;360:783-789.

Matthews DR, Charbonnel BH, Hanefeld M, et al. Long-term therapy with addition of pioglitazone to metformin compared with the addition of gliclazide to metformin in patients with type 2 diabetes: a randomized, comparative study. Diabetes Metab Res Rev. 2005;21:167-174.

Meriden T. Progress with thiazolidinediones in the management of type 2 diabetes mellitus. Clin Ther. 2004;26:177-190.

Mirkolf $\mathrm{H}$, Luebben G, Pfuetzner A, et al. Pioglitazone vs glibenclamide: significant differences in glycemic control and treatment failure rates in patients with type 2 diabetes mellitus. Presented at: 66th Scientific Sessions of the American Diabetes Association; June 9-13, 2006; Washington, DC. Abstract $n 604 \mathrm{p}$.

MRC/BHF (Medical Research Council/British Heart Foundation). Heart Protection Study of cholesterol-lowering therapy and of antioxidant vitamin supplementation in a wide range of patients at increased risk of coronary heart disease death: early safety and efficacy experience. Eur Heart $\mathrm{J}$. 1999;20:725-741.

National Service Framework for Diabetes. Standards 2001. Available at: www.doh.gov.uk/nsf/diabetes/ (accessed August 2007).

Nathan DM, Buse JB, Davidson MB, et al. Management of hyperglycemia in type 2 diabetes: A consensus algorithm for the initiation and adjustment of therapy: a consensus statement from the American Diabetes Association and the European Association for the Study of Diabetes. Diabetes Care. 2006;29:1963-1972.

Negro R, Dazzi D, Hassan $\mathrm{H}$, et al. Pioglitazone reduces blood pressure in nondipping diabetic patients. Minerva Endocrinol. 2004;29:11-17. 
Negro R, Mangieri T, Dazzi D, et al. Rosiglitazone effect on blood pressure and metabolic parameters in nondipper diabetic patients. Diabetes Res Clin Pract. 2005;70:20-25.

Nesto RW, Bell D, Bonow RO, et al. Thiazolidinedione use, fluid retention and congestive heart failure. A consensus statement from the American Heart association and American Diabetes Association. Diabetes Care. 2004;27:256-263.

Nissen SE, Wolski K. Effect of rosiglitazone on the risk of myocardial infarction and death from cardiovascular causes. N Engl J Med. 2007;356:2457-2471.

Oerter E, Lippmann-Grob, Luebben G. Pioglitazone vs glibenclamide: focus on metabolic control and health economic impact. Presented at: 66th Scientific Sessions of the American Diabetes Association; June 9-13, 2006; Washington, DC. Abstract 539-P.

Perez A, Khan M, Johnson T, et al. Pioglitazone plus a sulphonylurea or metformin is associated with increased lipoprotein particle size in patients with type 2 diabetes. Diab Vasc Dis Res. 2004;1:44-50.

Perez A, Spanheimer R, Kupfer S, et al. Cardiovascular safety profile of pioglitazone vs glyburide: results from a 3-year randomized, double blind trial. Presented at: 66th Scientific Sessions of the American Diabetes Association; June 9-13, 2006; Washington, DC. Abstract 1994-PO.

Peters Harmel AL, Kendall DM, Buse JB, et al. Impact of adjunctive thiazolidinedione therapy on blood lipid levels and glycemic control in patients with type 2 diabetes. Curr Med Res Opin. 2004;20:215-223.

Qayyum R, Adomaytyte J. A meta-analysis of the effects of thiazolidinediones on blood pressure. $J$ Clin Hypertens. 2006;8:19-28.

Reaven G. Role of insulin resistance in human disease. Diabetes. 1988;37:1595-1607.

Rosak $C$. The pathophysiologic basis of efficacy and clinical experience with the new oral antidiabetic agents. J Diabetes Complications. 2002;16:123-132.

Schernthaner G, Matthews DR, Charbonnel B, et al. Efficacy and safety of pioglitazone versus metformin in patients with type 2 diabetes mellitus: a double-blind, randomized trial. J Clin Endocrinol Metab. 2004;89:6068-6076.

Seufert J. A fixed-dose combination of pioglitazone and metformin: a promising alternative in metabolic control. Curr Med Res Opin. 2006;22(Suppl. 2):s39-s48.
Sharma PK, Bhansali A, Sialy R, et al. Effects of pioglitazone and metformin on plasma adiponectin in newly detected type 2 diabetes mellitus. Clin Endocrinol. 2006;65:722-728.

Singh S, Loke YK, Furberg CD. Long term risk of cardiovascular events with rosiglitazone. A meta-analysis. JAMA. 2007;298:1189-1195.

Spanheimer R, Perez A, Kupfer S, et al. The effects of pioglitazone vs glyburide on glycemic control in patients with type 2 diabetes in a 3-year randomized double-blind trial. Presented at: 66 th Scientific Sessions of the American Diabetes Association; June 9-13, 2006; Washington, DC. Abstract 320-OR

Standl E, Fuchtenbusch M. The role of oral antidiabetic agents: why and when to use an early phase insulin secretion agent in type 2 diabetes mellitus. Diabetologia. 2003;46(Suppl. 1):M30-M36.

Takiya L, Chawla S. Therapeutic options for the management of type 2 diabetes mellitus. Am J Manag Care. 2002;8:1009-1023.

Umpierrez G, Issa M, Vlajnic A. Glimepiride versus pioglitazone combination therapy in subjects with type diabetes inadequately controlled on metformin monotherapy: results of a randomized clinical trial. Curr Med Res Opin. 2006;22:751-759.

UKPDS (UK Prospective Diabetes Group). Effect of intensive blood glucose control with metformin on complications in overweight patients with type 2 diabetes (UKPDS 34). Lancet. 1998;352:854-865.

van Wijk JP, de Koning EJ, Martens EP, et al. Thiazolidinedones and blood lipids in type 2 diabetes. Arterioscler Thromb Vasc Biol. 2003;23:1744-1749. White JR, Davis SN, Cooppan R, et al. Clarifying the role of insulin in type 2 diabetes management. Clin Diabetes. 2003;21:14-21.

Correspondence: Prof Giuseppe Derosa, Department of Internal Medicine and Therapeutics, University of Pavia, Fondazione IRCCS Policlinico San Matteo, P. le Golgi 19, 27100 Pavia, Italy or at giuseppe.derosa@unipv.it 